\title{
Students' use of conceptual resources for understanding superposition
}

\author{
Lauren C. Bauman, \\ Quest University, 3200 University Blvd., Squamish, B.C., Canada, V8B 0N \\ Lisa M. Goodhew, ${ }^{1}$ and Amy D. Robertson ${ }^{2}$ \\ ${ }^{1}$ Department of Physics, University of Washington, Box 351560, Seattle, WA, USA, 98195-1560 \\ ${ }^{2}$ Department of Physics, Seattle Pacific University, 3307 Third Ave W, Seattle, WA, USA, 98119-1997
}

In this paper, we report the preliminary results of an investigation into introductory physics students' conceptual resources for understanding the principle of superposition. We analyzed 368 written responses to a conceptual question that asked students about situations for which the principle of superposition is useful/relevant. We identified four recurring resources related to superposition: (1) localization; (2) independence; (3) quantifiability; and (4) additiveness. Our objective is to support educators by drawing attention to these resources and by suggesting how they can be taken up alongside students to enhance instruction. 


\section{INTRODUCTION}

Pedaogical content knowledge (PCK), as introduced by Shulman [1], refers to "dimensions of subject matter knowledge for teaching." PCK can be thought of as a bridge between content knowledge (knowledge of subject matter) and pedagogical knowledge (knowledge of teaching). According to Shulman, PCK includes both knowledge of content and knowledge of student ideas (KSI). Frequently in physics education, research on student ideas, which informs instructors' KSI, focuses on identifying incorrect ideas or misunderstandings that prevent a student from answering questions correctly [2,3]. Research on student ideas about superposition follows this trend in its focus on student misunderstandings or difficulties. Another approach to KSI is to focus on resources: ideas that could represent the beginnings of more sophisticated scientific understandings [4-6]. Within the field of PER, few studies have taken a resources-based lens to identify common student ideas.

As superposition is central to various physical phenomena from pulses on a string to electric fields, it has been the focus of numerous studies about students' conceptual understanding. Such studies suggest that the principle of superposition causes difficulty for students [711]. Some commonly cited patterns of reasoning include: treating superposed pulses as objects that interact during a collision $[8,10]$, applying the principle of superposition at only the peaks of the pulses, or assuming that the peaks have to overlap for pulses to superpose [8]. Other research cites more general difficulties in applying superposition across contexts, particularly to electric fields $[7,9,11]$.

These examples highlight contributions to KSI in terms of prevalent misconceptions or difficulties, ideas that are incorrect and impede students' learning of physics. Our work adds to instructors' KSI by identifying prevalent resources for superposition, or student ideas that we see as continuous with formal physics understandings. Ideas that are continuous with formal physics are those that we, as physicists, see as having the potential to be generative for students. Ideas on a continuum with scientific thinking are not necessarily correct or complete but are "seeds" that could develop towards a canonically consistent physics understanding, help students solve problems, or support engagement in rich disciplinary practise.

Our analysis of students' written responses to a conceptual question about superposition (Fig. 1) resulted in our identification of four resources for understanding superposition: localization, independence, quantifiability, and additiveness. We contend that these resources represent elements of a scientific understanding of superposition. In reporting these resources, we aim to give instructors insight into some of the resources that students may use as they learn physics and suggest productive ways these ideas can be taken up with students to enhance instruction.

\section{THEORETICAL FRAMEWORK}

Our work is situated in resources theory, a "knowledge in pieces" approach to learning $[12,13]$. We define resources as elements of reasoning that could represent the beginnings of more sophisticated scientific understandings [4-6]. Resources are thought to be context-dependent and dynamically activated; the resources framework highlights the sometimes-incoherent and fluctuating nature of thinking [13]. That is, a resource activated for one question may not be activated for another, even if the two questions appear quite similar to experts. This understanding of student thinking aligns with that embodied by diSessa [14]: students have sensible ideas about physics that are useful in some contexts; students' thinking is often continuous with formal physics; and quality learning focuses on reorganizing and refining, rather than replacing, already present ideas.

The primary focus of our work is to support instructors by adding to their KSI "inventory" for superposition. Consequently, we use a model of generalizability that emphasizes recurrence across multiple sources of heterogeneity. We assume that if we see a pattern reproduced across contexts, we may be likely to see it again in similar contexts [15]. (Limitations to generalizability are discussed in the next section.)

\section{CONTEXT, QUESTION, \& ANALYSIS}

In this study, we analyzed 368 student responses to the examples of superposition question (Fig. 1). We designed this question to probe students' ideas about the nature of superposition, including the criteria that students use to determine when the principle applies. Students from two universities in the Pacific Northwest US-University of Washington (UW) and Western Washington University (WWU) - answered the question on homework, quizzes, or exams. In all cases, at least $70 \%$ of each sample responded.

The racial/ethnic demographics and parental income statistics for the universities in our study (blue bars) versus all college students (orange bars) are shown in Figures 2 and 3 . Orange bars were constructed from percentages cited in Kanim and Cid [16]; blue bars in Fig. 2 were constructed from university-level data reported by the Equality of Opportunity Project [17], weighted by sample size; blue bars in Fig. 3 were constructed from publicly-accessible

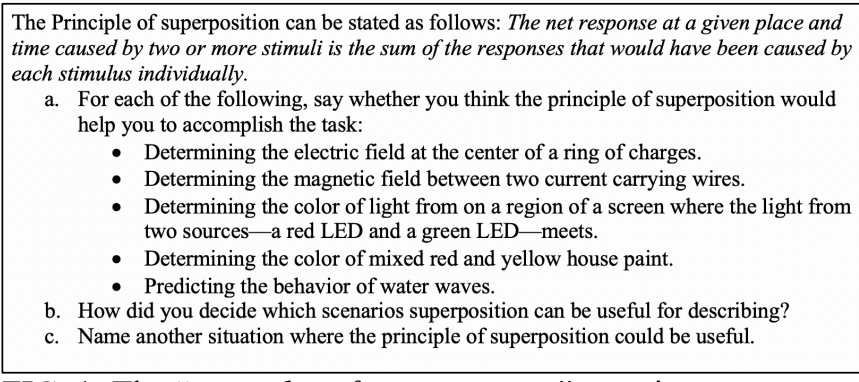

FIG. 1: The "examples of superposition" question. 
university-level demographic data [18,19], weighted by sample size. Both figures indicate that our research samples from universities that disproportionately serve white, wealthy students; this is aligned with a general (and problematic) trend in PER, as highlighted by Kanim and Cid. This imbalance limits the generalizability of our results, and the broader trend leads to a skewed accrual of the benefits of PER to white wealthy students. One limitation of Figures 2 and 3 is that they are based on university-level data, rather than data at the level of our sample (which we do not have); this assumes that the samples are demographically representative of the university. We are skeptical of this assumption and have made this a subject of future work.

The examples of superposition question asked students to determine whether the principle of superposition would be useful for thinking about five physical scenarios. The question also asks students to justify their responses and present their own (new) scenario where superposition could be useful. In our analysis, the first two and fifth scenarios are examples where superposition would be useful to accomplish the tasks; electric and magnetic fields add at points in space, and the amplitudes of individual water waves add to create a net response. Superposition would not be useful for the fourth scenario, paint mixing; pigments do not add in the same way that light does. The third scenario, determining the color of light, is less straightforward. One could argue superposition may be helpful since light waves obey superposition; however, human perception of colored light involves many interrelated phenomena that make this determination less clear.

Our analysis focused primarily on students' justifications. We developed an emergent coding scheme [20] by individually analyzing a subset of student responses for ideas that the authors understood as continuous with the principle of superposition. After individual analysis, we discussed patterns in the preliminary resources we identified and grouped similar ideas together. This process produced a final coding scheme of four resources.

Authors LCB and LMG independently coded student responses to part (b) of the examples of superposition question using this scheme. Resources can be - and often are-activated in concert; thus, a response could receive no code, one code, or multiple codes. We measured our percentage agreement by taking the normalized difference of all possible codes that could be assigned to a response, minus the number of coding disagreements of that response:

\section{$\underline{\left(n_{\text {possible codes }}\right)\left(n_{\text {coded responses }}\right)-n_{\text {coded disagreements }}}$ $\left(n_{\text {possible codes }}\right)\left(n_{\text {coded responses }}\right)$}

We used percentage agreement instead of other standard statistical measures of agreement (e.g., Cohen's kappa) because our codes are not independent or mutually exclusive [21]. Our percentage agreement for the full data set was $93 \%$.

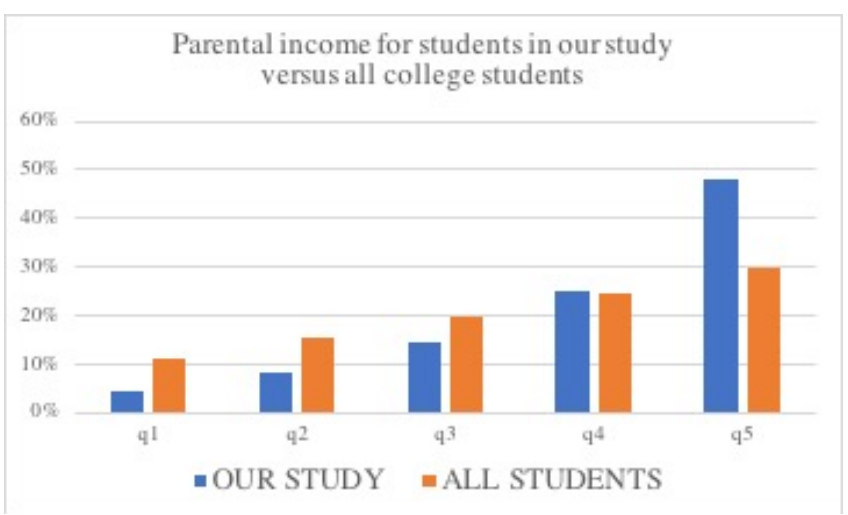

FIG. 2: Parental income for students in our study versus all college students. The Equality of Opportunity Project reports parental income by quintiles, so "q1" represents "quintile 1," or the bottom quintile.

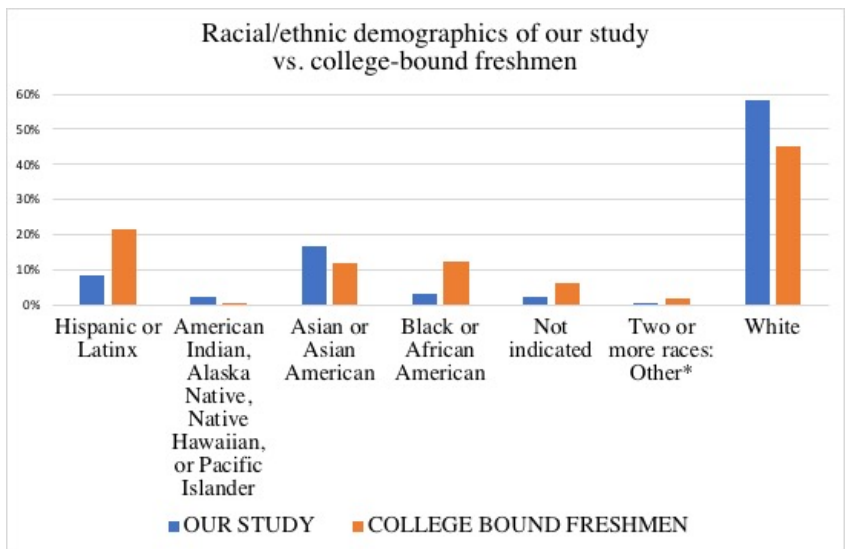

FIG. 3: Racial/ethnic demographics of our study versus college-bound freshmen. *UW does not report the demographic category "two or more races."

\section{RESOURCES}

As defined in the examples of superposition question, the principle of superposition states that the net response at a given place and time caused by two or more stimuli is the sum of the responses that would have been caused by each stimulus individually. Student responses to the examples of superposition question reflected elements of this definition. Our coding scheme highlights ideas that align with various elements of a "formal physics" understanding of superposition. In particular, we identified four resources in student responses that helped us to construct the following statement of the principle of superposition, which will ground our discussion throughout this section: Superposition applies to physical sources' effects that are localizedexisting in the same place at the same time; independentnot affecting each other; mathematizable, such that they can be quantified; and combined additively, contributing to a net effect. In this section, we will expand on each resource. 


\section{A. Localization: Superposed effects must be co-located.}

The localization resource expresses that superposition requires that effects be co-located in space and time. We assigned this code to student responses that emphasized any spatial or temporal co-occurrence. For example, one student determined that superposition applied to all of the examples listed in the question (Fig. 1), and justified their choice by saying, "All these scenarios required overlapping." Although this student's response to part (a) of the examples of superposition question was not completely correct, we saw their justification as continuous with the formal physics notion that superposed effects much be co-located. By limiting the application of superposition to scenarios where "overlapping" occurs, this response suggests the effects must be overlaid, or existing in the same place. Another example of the localization resource includes responses that explicitly stated a spatial and temporal co-location: "[Superposition applies to] any scenario that had multiple stimuli at a given place and time." We also observed many responses that suggested localization using similar descriptors like "intersecting," "point of intersection," "summing at one place," "where stimuli meet." All these examples acknowledge some spatial co-location-

\section{B. Independence: Effects must be independent of each other.}

This resource expresses the condition that superposed effects remain independent of each other, even though they are co-located in space. Conceptually, two sources do not change one another's effect, even though all sources change the net effect. We notice students expressing this notion in their justifications. For example, one student indicated that superposition applied to all scenarios except determining the color of mixed paint, and reasoned that "...the sum can be seen as a collection of individual components that don't alter each other." This response expresses the effects as "individual" and "not altering each other," indicating the notion of independence. Other responses which were assigned this code stressed the effects as "distinct" or "separate." One student described superposition as "useful for splitting quantities into components." We assigned the independence code to this response because to split a quantity into its components they must not change each other when combined.

\section{Quantifiability: Superposed effects can be described quantitatively.}

This resource emphasizes superposition as a measurable and mathematizable phenomenon. Many students used quantifiability as a condition by which they determined situations in which the principle of superposition applies. We coded any responses which emphasized the importance of mathematizing, measuring, accounting or assigning numbers or vectors to variables as "quantifiability." For example, one response read: "Superposition is useful and applies to scenarios where each stimulus has a quantitative, measurable effect. It isn't useful with colors because colors are qualitative and can't be "summed." This example also illustrates how quantifiability motivates the "additiveness" of superposition, which we unpack below: the student argues that without quantifiable inputs there is nothing to sum. A number of students used a similar line of reasoning to justify why superposition is not helpful for mixing paint.

\section{Additiveness: Effects add together.}

Additiveness is foundational to superposition; it expresses what superposition is: the summation of two effects to find a net effect. We assigned this code to responses that indicated that superposition requires adding, finding the net effect, summing, or combining effects. Some students directly referenced adding. For example, one student said that superposition applies to all the scenarios listed and wrote, "They are additive (two or more sources)." Other students described the process of addition without using the word itself. For example, one student wrote, "If input $\mathrm{A}$ produces response $\mathrm{X}$ and input $\mathrm{B}$ produces response $\mathrm{Y}$, then input $(\mathrm{A}+\mathrm{B})$ produces response $(\mathrm{X}+\mathrm{Y})$."

\section{PREVALENCE OF RESOURCES}

As we have described, the purpose of this project is to identify common, prevalent resources, with the intention of providing instructors with high-leverage, pragmatic KSI. In previous work, we have considered a resource "common" if it appeared: (a) in responses to multiple questions in our study and (b) in at least 10\% of student responses for at least one sample [22]. Because this study is preliminary, we have only identified resources in student responses to one question (examples of superposition). Thus, we can only determine the commonality of these resources within the context of that question. We report the prevalence of each resource to show that they are not idiosyncratic and to show which resources tend to be common in the context of this question.

As reflected in Table I, the quantifiability and additiveness resources were most common, with between $5 \%$ and $30 \%$ of students using the former and $29 \%$ to $42 \%$ of students using the latter. The prevalence of these resources

TABLE I. Prevalence of each resource. Percentages represent responses in which both coders were in agreement.

\begin{tabular}{cccc}
\hline \hline \multirow{2}{*}{ Resources } & \multicolumn{3}{c}{ Prevalence } \\
& WWU & UW & Overall \\
& $\mathrm{n}=206$ & $\mathrm{n}=162$ & $\mathrm{n}=368$ \\
\hline Localization & $5 \%$ & $9 \%$ & $7 \%$ \\
Independence & $2 \%$ & $7 \%$ & $5 \%$ \\
Quantifiability & $5 \%$ & $30 \%$ & $16 \%$ \\
Additiveness & $29 \%$ & $42 \%$ & $35 \%$ \\
\hline \hline
\end{tabular}


in this question leads us to speculate that these resources may be used by students in other, similar contexts, with noted limitations based on our sample demographics.

The localization and independence resources were less common, represented in only $2 \%$ to $9 \%$ of responses (see Table I). Although recurrent (across two samples), these resources would not be considered "common" based on the definition laid out in our previous work [18]. However, we chose to include them here because we see them as being useful elements of knowledge that contribute to an understanding of superposition and thus that instructors might pay attention to and build on when they do emerge in instruction, even if infrequently. Further, this study is preliminary and these resources were not investigated in the context of multiple questions. It is possible that the localization and independence resources would be more commonly activated in other contexts. For example, Sengören et al. [8] report ideas that resemble the localization resource in the context of a question about superposition of mechanical pulses.

\section{IMPLICATIONS FOR INSTRUCTION}

Our work is motivated by our efforts to add to instructors' KSI repertoire in the context of superposition by (a) presenting prevalent resources amongst students and (b) suggesting ways in which these resources could be taken up alongside students to enhance instruction. In this section we address the latter by discussing two ways these resources could be used to enhance instruction.

\section{A. Eliciting resources for superposition}

Past research suggests there are many contexts in which students have difficulty applying superposition, particularly in questions about electric fields [7,9,11]. The analysis we present here suggests that students possess sophisticated and potentially fruitful ideas about superposition that can be elicited in particular contexts. In our efforts to identify student resources for superposition, we observed many responses that are aligned with various pieces of a formal definition of superposition. For example, students used the resources localization, independence, quantifiability, and additiveness to justify why superposition does or does not apply to several example scenarios. These resources express that effects must be co-located in space, independent of one another, able to be expressed as a quantity, and summable, all pointing to something fundamental about superposition. In other words, the superposition examples question is one context that appears to elicit a variety of resources for understanding superposition. Instructors may wish to try this question in their own instruction, and then take up these resources by working alongside students to refine and apply their existing ideas about superposition to new contexts. Future work could focus on identifying additional contexts and questions that elicit these ideas for many students.

\section{B. Reframing student ideas}

As previously presented, past research suggests students have difficulty applying the principle of superposition. For example, Sengören et al. [8] identified student difficulties in applying superposition to waves; the authors say that students often argue that "[t]he superposition principle cannot be applied unless the peaks of the pulses overlap." An instructor approaching this idea from a resource perspective might seek to identify aspects of students' reasoning that are continuous with formal physics. For example, we would interpret the statement "superposition applies when pulses overlap" as using the localization resource because "overlapping" suggests the effects must be overlaid, or existing in the same place. Instead of framing instruction as an opportunity to replace the student's incorrect conception (the peaks of the pulses must overlap) with correct theory, an instructor could work with the student to refine and reorganize their already present ideas. In a resources perspective, the student's underlying thinking (superposition applies when there is overlap) is continuous with formal physics; however, the idea may need refinement to account for all places where two or more effects are co-located. This re-framing of students' thinking highlights the disciplinarilysophisticated ideas students already possess.

\section{LIMITATIONS}

The four conceptual resources presented here add to previous PER research that identifies difficulties or misconceptions about superposition. As we discussed in Section III, our analysis of student responses was informed by the resources theoretical framework; we looked for student ideas that we identified as continuous with formal physics. In this sense, the resources we identified in our data were shaped by our own understandings of superposition and by the orientation toward student ideas that is embodied by resources theory. A researcher with different commitments and/or understandings may well see different interpretive possibilities in this data. Our effort here has been to describe the categories that we inferred, expecting that others may be able to "f[i]nd or recogniz[e]" these categories "once [they] ha[ve] been described to them by the original researcher[s]" (us) [23]. It is not to suggest that "other researchers [would] find the same conceptions or categories if they were doing the study for the first time" [23].

\section{ACKNOWLEDGEMENTS}

This work was supported in part by the National Science Foundation under grant numbers 1608510, 1608221, and 1256082. The authors wish to thank Paula R. L. Heron and Rachel E. Scherr for offering helpful feedback, Ian M. Hoffman for being a sounding board, and Andrew Boudreaux for helping with data collection. 
[1] L. Shulman, Harv. Educ. Rev. 57, 1 (1987).

[2] L.C. McDermott, and E.F. Redish, Am. J. Phys. 67, 9 (1999).

[3] D.E. Brown, and D. Hammer, in International Handbook of Research on Conceptual Change, edited by S. Vosniadou (Routledge, New York, NY, 2008), p. 127-154.

[4] D. Hammer, Am. J. Phys. 69, S52 (2000).

[5] D. Hammer, and E. van Zee, Seeing the Science in Children's Thinking: Case Studies of Student Inquiry in Physical Science (Heinemann, Portsmouth, NH, 2006).

[6] J.P. Smith, A.A. diSessa, and J. Roschelle, J. Learn. Sci. 3, 2 (1993).

[7] J. Li, and C. Singh, Eur. J. Phys. 38, 055702 (2017).

[8] S.K. Sengören, R. Tanel, and N. Kavcar, Phys. Educ. 41, 453 (2006).

[9] C. Singh, Am. J. Phys. 74, 923 (2006).

[10] M.C. Wittmann, R.N. Steinberg, and E.F. Redish, Phys. Teach. 37, 15 (1999).

[11] S. Rainson, G. Transtromer, and L. Viennot, Am. J. Phys. 62, 11 (1994).
[12] D. Hammer, A. Elby, R.E. Scherr, and E.F. Redish, in Transfer of Learning from a Modern

Multidisciplinary Perspective, edited by J.P. Mestre (Information Age Publishing, Greenwich, 2005), p. 89-119.

[13] R.E. Scherr, Am. J. Phys. 75, 272 (2007).

[14] A. A. diSessa, Cognition Instruct. 10, 2 \& 3 (1993).

[15] T.D. Cook, Educ. Eval. Policy An. 24, 3 (2002).

[16] S. Kanim, X.C. Cid, The demographics of physics education research (2017)

[17] https://equality-of-opportunity.org/data. Retrieved $12 / 05 / 2018$.

[18] https://www.wwu.edu/diversity. Retrieved 09/27/2019.

[19] https://studentdata.washington.edu/wpcontent/uploads/sites/3/2018/10/Quick_Stats_Seattle_ Aut2018.pdf. Retrieved 09/27/2019.

[20] K. Krippendorff, Content Analysis: An Introduction to Its Methodology 3rd ed. (Sage, Thousand Oaks, 2013)

[21] M. Banerjee, M. Capozzoli, L. McSweeney, and D. Sinha, Can. J. Stat. 27, 1 (1999).

[22] L.M. Goodhew A. D. Robertson, P. R. L. Heron, and R. E. Scherr, Phys. Rev. PER (under revision).

[23] F. Marton, J. Thought 21, 28 (1986). 